\title{
A novel subpopulation of monocyte-like cells in the human lung after lipopolysaccharide inhalation
}

\author{
Mairi Brittan*, Laura Barr*, Andrew Conway Morris*, Rodger Duffin*, \\ Fiona Rossi*, Shonna Johnston*, Graham Monro*, Niall Anderson* , Adriano G. Rossi*, \\ Danny F. McAuley ", Christopher Haslett*, Nik Hirani*, Kev Dhaliwal* and \\ A. John Simpson*,+
}

ABSTRACT: The co-ordinated recruitment of monocyte subpopulations, neutrophils and regulatory T-cells (Tregs) during the early stages of human acute lung inflammation remains poorly understood. We therefore performed a detailed characterisation of these lineages in the blood and lungs in a model of human acute lung inflammation.

Healthy volunteers inhaled lipopolysaccharide (LPS) or saline ( $n=6$ for each group). Blood was collected at 0, 2, 4, 6 and $8 \mathrm{~h}$ and bronchoscopy with bronchoalveolar lavage (BAL) performed at $8 \mathrm{~h}$. Multiparameter flow cytometry was used to characterise monocyte subpopulations, neutrophils and Tregs in the blood and lung.

Inhalation of LPS was associated with significant blood and BAL fluid neutrophilia. Blood populations of monocyte subpopulations and Tregs were unaltered by LPS. In contrast, LPS induced an accumulation of a pulmonary monocyte-like cell (PMLC) population, which was further subdivided into "inducible" CD14 ${ }^{++} \mathrm{CD} 16$ " and "resident" CD14 ${ }^{++} \mathrm{CD} 16^{+}$subsets. Inducible PMLCs were significantly increased following LPS inhalation $(p=0.0046)$, whereas resident PMLCs were unchanged. In addition, we noted a significant decrease in Tregs in BAL fluid with LPS inhalation $(p=0.027)$.

The early stages of LPS-induced inflammation in humans is characterised by pulmonary accumulation of a novel inducible monocyte-like subpopulation, accompanied by significant changes in both neutrophil and Treg numbers.

KEYWORDS: Acute lung inflammation, macrophages, monocytes, multiparameter flow cytometry, regulatory $\mathrm{T}$-cells

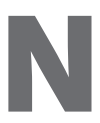
eutrophils are considered the archetypal cell type in the acute inflammatory response, and are frequently the primary focus of studies of inflammatory lung diseases. Neutrophils are rapidly recruited to sites of infection and tissue injury where they accumulate to destroy and digest invading pathogens, before undergoing apoptosis and clearance by macrophages. Prolonged neutrophil lifespan and dysregulated macrophage clearance of apoptotic neutrophils contribute to the pathogenesis of inflammatory and fibrotic lung disease [1]. Evidence from animal studies has highlighted reciprocal, interdependent relationships between monocytes and neutrophils during the early inflammatory response. For example, monocytes are critical regulators of the extravasation of neutrophils from the pulmonary blood vessels and their infiltration into the inflamed mouse lung $[2,3]$. Regulatory T-cells (Tregs) are reduced in the lungs of patients with chronic obstructive pulmonary disease (COPD) [4], and it is proposed that Tregs play important roles during the resolution of lung inflammation [5]. However, the co-ordinated actions of monocytes, neutrophils and Tregs in the pathogenesis of human lung inflammation remain poorly understood.

A blood monocyte-derived cell with heightened pro-inflammatory mediator secretion has previously been described in the human lung in sarcoidosis [6, 7], idiopathic pulmonary fibrosis (IPF) $[7,8]$ and HIV-related pulmonary disease [9]. Similarly, an immature "alveolar monocyte" was described in bronchoalveolar lavage (BAL)
AFFILIATIONS

*University of Edinburgh, MRC Centre for Inflammation Research, The Queen's Medical Research Institute,

${ }^{\#}$ Centre for Population Health Sciences, The University of Edinburgh Medical School, Edinburgh,

"Respiratory Medicine Research Cluster, Centre for Infection and Immunity, Microbiology Building, The Queen's University of Belfast, Belfast, and

${ }^{+}$Institute of Cellular Medicine, Medical School, Newcastle University, Newcastle upon Tyne, UK.

\section{CORRESPONDENCE}

M. Brittan

University of Edinburgh

MRC Centre for Inflammation

Research

The Queen's Medical Research

Institute

47 Little France Crescent

Edinburgh

EH16 4TJ

UK

E-mail: mbrittan@staffmail.ed.ac.uk

Received:

July 042011

Accepted after revision:

Oct 222011

First published online:

Jan 202012

European Respiratory Journal

Print ISSN 0903-1936

Online ISSN 1399-3003 
fluid from lungs of patients with acute respiratory distress syndrome, with increasing numbers correlating with disease severity [10]. However, since these early reports, few studies have investigated monocytes and their progeny in the human lung, and their phenotype, function and interactions with other leukocyte populations are not known.

We have performed an extensive characterisation of human monocyte subsets, neutrophils and Tregs in peripheral blood and cells retrieved by BAL in healthy volunteers exposed to bacterial lipopolysaccharide (LPS), or saline control. Inhalation of LPS by healthy human volunteers induces experimental blood and lung inflammation similar to the early stages of acute pulmonary inflammation, without significant adverse effects [11-16].

We report, for the first time, the presence of a human pulmonary monocyte-like cell (PMLC) with distinct "resident" (rPMLC) and "inducible" (iPMLC) subpopulations in BAL fluid (BALF) at $8 \mathrm{~h}$ post-LPS inhalation. The iPMLC subpopulation is significantly increased by LPS compared with saline, in parallel with a significant increase in pulmonary neutrophils and a significant decrease in pulmonary Tregs.

\section{MATERIALS AND METHODS Subjects}

Male volunteers aged 18-40 yrs were recruited. Exclusion criteria consisted of: ongoing acute or chronic illness; current medication; history of smoking within the previous year or previous smoking history amounting to $>2$ pack-yrs; alcohol intake $>21$ units per week; abnormal findings on cardiorespiratory examination or chest radiograph; forced expiratory volume in $1 \mathrm{~s}$ (FEV1) or forced vital capacity (FVC) $<80 \%$ of predicted or FEV1/FVC ratio $<70 \%$; or abnormal routine blood haematology/ biochemistry. This study was approved by the Lothian research ethics committee and the NHS Lothian/University of Edinburgh Research and Development Office, Edinburgh, UK. Written informed consent was obtained from all subjects.

\section{Study design}

Volunteers were randomly assigned to inhalation of LPS from Escherichia coli, dissolved in endotoxin-free sterile $0.9 \%$ saline, or saline placebo, in a single-blinded manner ( $n=6$ for each group). A dose of $60 \mu \mathrm{g}$ LPS was administered using an inhalationsynchronised dosimeter nebuliser (Spira Elektro 2, Hameenlinna, Finland) calibrated to produce an aerosol of $9.6 \mu \mathrm{L}$ over $0.6 \mathrm{~s}$ with five slow inhalations from functional residual capacity. This dose of LPS was selected in order to reliably elicit reproducible inflammation both systemically and within the lung, while keeping the risk of adverse events to a minimum [14,17]. Routine clinical observations were recorded hourly. Venous blood samples were collected into EDTA at baseline (0), 2, 4, 6 and $8 \mathrm{~h}$ following LPS/saline inhalation. BAL of the right middle lobe was performed at $8 \mathrm{~h}$, as previously described [18].

\section{Full blood counts}

Automated full blood counts were performed by the Royal Infirmary of Edinburgh Clinical Haematology Laboratory, Edinburgh, UK, on a venous blood sample drawn at each time-point.

\section{Processing of BALF}

Total white cell count in BALF was determined using a haemocytometer. Cell viability was determined by trypan blue staining; total and nonviable cell counts were confirmed by an automated NucleoCounter (ChemoMetec, Allerød, Denmark) using cartridges pre-coated with propidium iodide [19]. BALF was filtered through a $40 \mu \mathrm{m}$ cell strainer, centrifuged at $200 \times g$ for $10 \mathrm{~min}$ at $4^{\circ} \mathrm{C}$ and supernatant stored at $-80^{\circ} \mathrm{C}$. Cells were resuspended in PBS and centrifuged as above, supernatant discarded and cells resuspended in $0.1 \%$ bovine serum albumin (BSA) in PBS with $\mathrm{Ca} / \mathrm{Mg}$ at a concentration of 1 million cells per $\mathrm{mL}$ for flow cytometry.

\section{Staining of blood and BALF for flow cytometry}

Flow cytometry protocols were largely based upon those of FUNG et al. [20]. Antibody mixtures for each staining panel were prepared at each time-point (table 1). Experimental controls for each individual (at baseline for blood and for BALF) consisted of single antibody stains, isotype controls, and fluorescence minus one (FMO) controls for CCR2 and CX3CR1 (all reagents minus the antibody of interest, which was substituted for a relevant isotype control) [21]. An unstained control was prepared for all blood and BALF samples. Antibodies were incubated with whole blood $(50 \mu \mathrm{L})$ or BALF $(100 \mu \mathrm{L})$ in a sterile polystyrene tube (352001, Becton Dickinson (BD Biosciences, Oxford, UK)) for $30 \mathrm{~min}$ on ice. Blood plus antibodies were incubated with $50 \mu \mathrm{L}$ flow cytometry staining buffer (00-4222-26; eBioscience, Hatfield, UK), and BALF plus antibodies were incubated with $100 \mu \mathrm{L}$ $0.1 \%$ BSA in PBS with magnesium and calcium. Erythrocytes were lysed by incubation in $750 \mu \mathrm{L}$ FACS lysing solution (349202; BD Biosciences, Franklin Lakes, NJ USA) at room temperature for $20 \mathrm{~min}$. Samples were washed in $2 \mathrm{~mL} \mathrm{PBS}$, centrifuged at $350 \times g$ for $5 \mathrm{~min}$, supernatants discarded and cells resuspended in $500 \mu \mathrm{L}$ FACS lysing solution (BD Biosciences). Flow cytometry was performed using a BD SORP LSRFortessa cell analyser using FACSDiva software (BD Biosciences). Data analyses were performed using FlowJo version 9.1 (TreeStar, Ashland, OR, USA). A minimum of 150,000 cells was captured for each sample. The flow cytometer was calibrated daily. Compensation was calculated automatically then verified manually, for each antibody using antimouse immunoglobulin (Ig) or anti-rat Ig BD ${ }^{\mathrm{TM}}$ CompBeads (552843, 552844; BD Biosciences). Voltages for each parameter remained consistent throughout the study.

Monocytes, neutrophils and lymphocytes in blood were gated on flow cytometry dot plots of forward scatter-area (FSC-A) versus side scatter-area (SSC-A). Monocytes were defined as $\mathrm{HLA}_{-\mathrm{DR}}{ }^{+}$and the proportion of classical $\left(\mathrm{CD} 14^{++} \mathrm{CD} 16^{-}\right)$, intermediate $\left(\mathrm{CD} 14^{++} \mathrm{CD} 16^{+}\right)$and nonclassical $\left(\mathrm{CD} 14^{+} \mathrm{CD} 16^{++}\right)$ monocyte subpopulations calculated as a percentage of total HLA-DR ${ }^{+}$monocytes. The expression of HLA-DR, CX3CR1, CCR2, CD11b, CD62L and CD64 by each monocyte subset was defined as high $\left({ }^{++}\right)$, intermediate $\left(^{+}\right)$or low/negative $\left(^{-}\right)$by calculating geometric mean fluorescence (GMF) and comparing GMF intensity for each antibody by each monocyte subset throughout the time course of LPS/saline administration. Similarly, expression of CD16, CD62L, CD11b and CD64 by neutrophils was compared throughout the LPS/saline time course. T-lymphocytes were identified as $\mathrm{CD}^{+}$cells within the lymphocyte gate, and CD25 $5^{+}$CD $127^{-}$Tregs were calculated as a percentage of total $\mathrm{CD}^{+} \mathrm{CD}^{+}$lymphocytes [22]. Distinct populations of PMLCs, neutrophils, lymphocytes and alveolar 
TABLE 1 Antibody staining panels used for flow cytometry

\begin{tabular}{|c|c|c|c|c|c|c|}
\hline Antibody & Host species, isotype & Clone & Fluorochrome & $\begin{array}{l}\text { Manufacturer and } \\
\text { catalogue number }\end{array}$ & Laser filter & Band-pass \\
\hline \multicolumn{7}{|c|}{ Monocyte and neutrophil panel } \\
\hline Anti-hCD14 & Mouse $\lg \mathrm{G} 1$ & HCD14 & PerCP/Cy5.5 & BioLegend 325622 & Blue & $695 / 40$ \\
\hline Anti-hCD16 & Mouse IgG1 & $3 G 8$ & PE/Texas Red & Invitrogen MHCD1617 & $\mathrm{Y} / \mathrm{G}$ & $610 / 20$ \\
\hline Anti-hCCR2 & Mouse lgG2b & 48607 & AF647 & BD Pharmingen 558406 & Red & $670 / 30$ \\
\hline Anti-hCD11b & Mouse lgG1 & ICRF44 & PE/Cy5 & BioLegend 301308 & $Y / G$ & $670 / 30$ \\
\hline Anti-hCD62L & Mouse IgG1 & DREG58 & APC/Cy7 & BioLegend 304814 & Red & $780 / 60$ \\
\hline Anti-hCD64 (FcrR1) & Mouse IgG1 & 10.1 & PE & BioLegend 305008 & $Y / G$ & $582 / 15$ \\
\hline \multicolumn{7}{|c|}{ Regulatory T-lymphocyte panel } \\
\hline Anti-hCD3 & Mouse IgG1 & UCHT1 & FITC & BD Pharmingen 555332 & Blue & $530 / 30$ \\
\hline Anti-hCD4 & Mouse IgG1 & RPA-T4 & $\mathrm{APC} / \mathrm{Cy} 7$ & BioLegend 300518 & Red & $780 / 60$ \\
\hline
\end{tabular}

h: human; Ig: immunoglobulin; PE: phycoerythrin; Y/G: yellow/green; FITC: fluorescein isothiocyanate; HLA: human leukocyte antigen; APC: allophycocyanin.

macrophages (AMs) were gated in BALF from LPS and salinetreated volunteers on FSC-A versus SSC-A flow cytometry dot plots. Antibody expression by each population was then assessed, as above.

\section{Cytokine measurement in BALF}

ELISA for interleukin (IL)-6, IL-8, monocyte-chemoattractant protein (MCP)-1 and tumour necrosis factor (TNF)- $\alpha$ was performed on supernatants from BALF according to the manufacturers' instructions (R\&D Systems, DuoSet ${ }^{\circ}$ ELISA Development Systems, Minneapolis, MN, USA).

\section{Statistical analyses}

Statistical analyses were performed using GraphPad Prism (version 4.0, GraphPad Software, Inc., San Diego, CA, USA). Normality of data was assessed using the modified KolmogorovSmirnov test. Data with a normal distribution were expressed as mean \pm SD and analysed using a two-sample unpaired t-test. Data that were not normally distributed were expressed as median (interquartile range) and analysed by Mann-Whitney U-test. A p-value of $<0.05$ was considered significant.

\section{Full blood counts}

To compare changes in circulating blood neutrophil counts between the saline and LPS groups, the mean neutrophil count between baseline and $8 \mathrm{~h}$ (equivalent to "area under the curve") was calculated for each volunteer. Mean neutrophil counts were then assessed for normality and analysed as described below [23]. The same method was used to compare changes in circulating blood monocyte, lymphocyte and platelet counts between the groups.

\section{Flow cytometry analyses}

The relative contribution of each monocyte subpopulation to total monocytes and of Treg cells to total $\mathrm{CD}^{+} \mathrm{CD}^{+} \mathrm{T}$ lymphocytes, in peripheral blood between 0 and $8 \mathrm{~h}$ in LPStreated compared to saline-treated subjects was analysed by two-way repeated measures analysis of variance (ANOVA), with post-test Bonferroni corrections. This enabled analysis of the effects of time, treatment (saline or LPS) and the combination of time and treatment on the proportion of each cell type. Differences in cell surface antigen expression (GMF intensity) by monocyte subsets and neutrophils for both groups were also analysed by this method.

\section{BAL cytokine concentrations}

The concentration of TNF- $\alpha$ in BALF from two out of five volunteers in the LPS-treated group, and from all saline-treated volunteers were below the lower limit of detection of the assay and therefore precise analysis was not possible. Concentrations of MCP-1 in BALF from the saline-treated group were all below the lower limit of detection of the assay $\left(15.62 \mathrm{pg} \cdot \mathrm{mL}^{-1}\right)$. To enable statistical comparison with MCP-1 concentrations in the LPS group, the saline group MCP-1 concentrations were therefore each assigned a ranked value lower than $15.62 \mathrm{pg} \cdot \mathrm{mL}^{-1}$.

\section{RESULTS}

The baseline characteristics of the LPS and saline groups were closely matched (table 2). Bronchoscopy and BAL was completed in 11 volunteers (one volunteer in the LPS group did not tolerate bronchoscopy). No serious adverse events were reported and all symptoms resolved within $24 \mathrm{~h}$. Five volunteers who inhaled LPS developed pyrexia, two reported cough and one myalgia. One volunteer who inhaled saline reported pyrexial symptoms after BAL. The LPS-treated group displayed a significantly higher maximum increment in temperature $\left(1.35 \pm 0.41^{\circ} \mathrm{C}\right.$ versus $0.75 \pm 0.50^{\circ} \mathrm{C}$; two-sample t-test, $\left.\mathrm{p}=0.047\right)$ and a significantly higher maximum increment in cardiac frequency ( $26 \pm 11$ versus $5 \pm 4$ beats $\mathrm{min}^{-1}$; two-sample t-test, $\mathrm{p}=0.0014$ ) compared with the saline-treated group. All FEV1 and FVC measurements were maintained within $10 \%$ of baseline when measured pre-bronchoscopy. Changes in FEV1 and FVC from baseline did not differ significantly between groups (mean change in FEV1 $-1.91 \pm 4.13 \%$ versus $+0.15 \pm 3.56 \%$, 


\begin{tabular}{lcc}
\hline TABLE 2 & $\begin{array}{l}\text { Baseline characteristics of the saline and } \\
\text { lipopolysaccharide (LPS) groups }\end{array}$ \\
Characteristic & Saline & LPS \\
\hline Subjects $\mathbf{n}$ & 6 & 6 \\
Age yrs & $24.7 \pm 5.1$ & $24.8 \pm 6.2$ \\
Ethnicity & $5 / 6$ Caucasian & $5 / 6$ Caucasian \\
Height $\mathbf{m}$ & $1.81 \pm 0.14$ & $1.77 \pm 0.04$ \\
Weight $\mathbf{k g}$ & $81.9 \pm 17.2$ & $79.6 \pm 20.7$ \\
Resting cardiac frequency beats min $^{-1}$ & $72.3 \pm 14.5$ & $69.0 \pm 10.1$ \\
Baseline FEV $\mathbf{1} \%$ pred & $107.5 \pm 9.0$ & $104.0 \pm 9.3$ \\
Baseline temperature ${ }^{\circ} \mathbf{C}$ & $36.3 \pm 0.3$ & $36.3 \pm 0.5$ \\
\hline & & \\
Data are presented as mean \pm SD, unless otherwise stated. FEV $1:$ forced \\
expiratory volume in $1 \mathrm{~S} ; \%$ pred: \% predicted.
\end{tabular}

two-sample t-test, $\mathrm{p}=0.38$; mean change in FVC $-2.10 \pm 4.11 \%$ versus $-2.09 \pm 2.91 \%$, two-sample t-test, $\mathrm{p}=0.99$ ).

LPS inhalation was associated with a significant peripheral blood neutrophilia, initially evident at $4 \mathrm{~h}$ and which peaked at 8 h (fig. 1; mean neutrophil count over time $5.12 \pm 1.10 \times 10^{9}$ per litre versus $2.61 \pm 0.95 \times 10^{9}$ per litre; two-sample t-test, $p=0.002)$. No significant difference was observed in the total numbers of circulating peripheral blood monocytes, lymphocytes or platelets in the LPS subjects compared with saline controls (data not shown).

The relative contribution of classical, intermediate or nonclassical monocyte subpopulations to total HLA-DR ${ }^{+}$monocytes in peripheral blood did not change over time in either the LPS or saline groups (fig. 2). Furthermore, no significant differences were observed in the relative contribution of any monocyte subpopulation to total monocyte number at any time, when comparing LPS and saline inhalation (fig. 2). The expression of

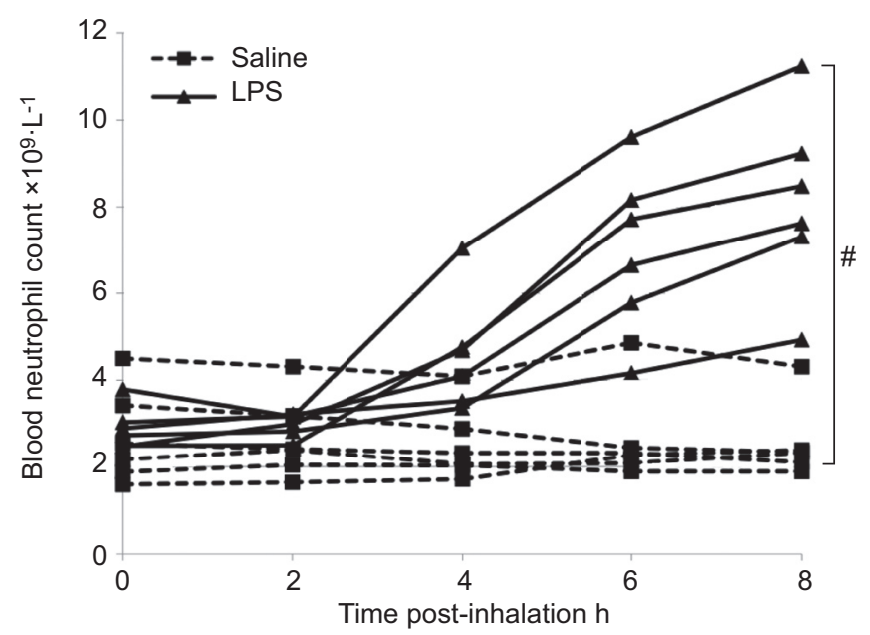

FIGURE 1. Circulating blood neutrophil counts following saline or lipopolysaccharide (LPS) inhalation. A significant rise in circulating blood neutrophil count was observed after inhalation of LPS (but not saline), which peaked at $8 \mathrm{~h}$. Mean neutrophil count between 0 and $8 \mathrm{~h}$ was significantly higher in the LPS group. ${ }^{\# \text { : }}$ $p<0.002$
HLA-DR, CX3CR1, CCR2, CD64, CD11b and CD62L by peripheral blood monocyte subpopulations remained consistent throughout the time course of LPS or saline administration (i.e. at each of five time-points, from each of 12 volunteers, GMF intensity of expression by each monocyte subpopulation
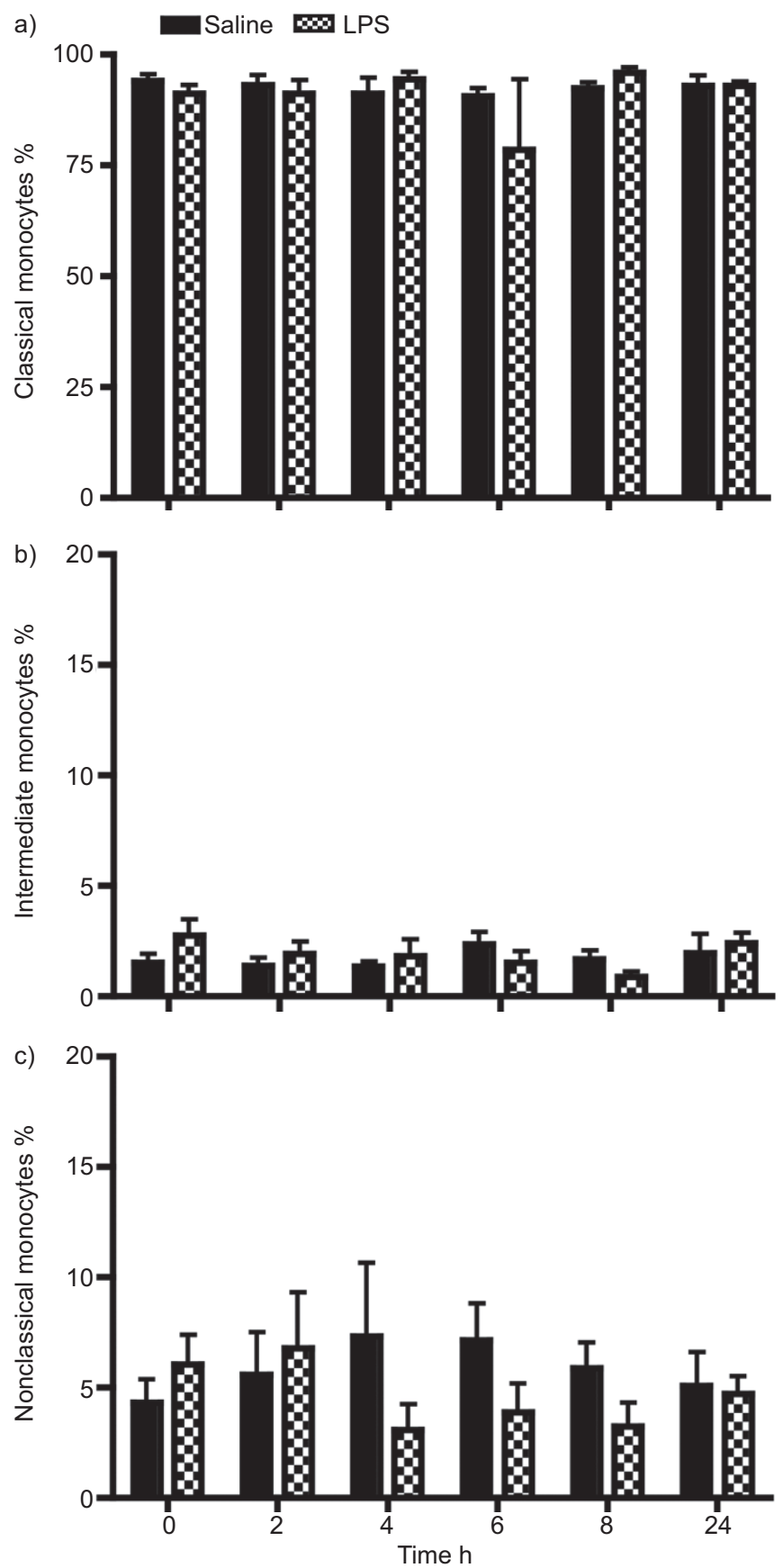

FIGURE 2. Relative contribution of a) classical, b) intermediate and c) nonclassical monocyte subsets to total human leukocyte antigen-DR ${ }^{+}$monocytes in peripheral whole blood, throughout the time course of lipopolysaccharide (LPS) or saline administration. No significant differences were observed between volunteers administered LPS compared with saline. Likewise, no significant differences were observed throughout the time course of either LPS or saline administration (two-way repeated measures ANOVA, columns represent means, error bars show standard error of the mean). 


\begin{tabular}{lccc} 
TABLE 3 & \multicolumn{3}{l}{$\begin{array}{l}\text { Cell surface marker expression by monocyte } \\
\text { subpopulations in peripheral blood }\end{array}$} \\
Antigen & $\begin{array}{c}\text { Classical } \\
\text { monocytes }\end{array}$ & $\begin{array}{c}\text { Intermediate } \\
\text { monocytes }\end{array}$ & $\begin{array}{c}\text { Nonclassical } \\
\text { monocytes }\end{array}$ \\
\hline HLA-DR & + & + & + \\
CD14 & ++ & + & + \\
CD16 & - & + & + \\
CCR2 & + & + & - \\
CX3CR1 & - & + & + \\
CD11b & ++ & + & + \\
CD62L & + & + & - \\
CD64 & ++ & + & - \\
\hline
\end{tabular}

HLA: human leukocyte antigen. ${ }^{*}$ : the expression of each antigen by each monocyte subset is presented as high $\left({ }^{++}\right)$, intermediate $\left(^{+}\right)$or low/negative $\left({ }^{(}\right)$. No significant differences were observed in geometric mean fluorescence intensity of expression for each antigen by each monocyte subpopulation over time or between saline and lipopolysaccharide groups.

remained high, intermediate or low/negative) (table 3). Moreover, the proportion of $\mathrm{CD}_{2} 5^{+} \mathrm{CD} 127^{-}$regulatory $\mathrm{T}$ lymphocytes (as a percentage of total $\mathrm{CD}^{+} \mathrm{CD} 4^{+}$lymphocytes) in peripheral blood did not change throughout the time course of LPS/saline administration. Figure 3 shows the gating strategies used for flow cytometric assessment of monocytes, neutrophils and Tregs in whole blood.

\section{Analysis of BALF}

The total number of cells per millilitre of BALF was greater in the LPS-treated volunteers compared with saline controls (mean cell count $3.12 \pm 1.35 \times 10^{5}$ per $\mathrm{mL}$ versus $1.10 \pm 0.35 \times 10^{5}$ per $\mathrm{mL}$; two-sample t-test, $\mathrm{p}=0.006)$. The mean percentage of nonviable cells was $<16 \%$ of the total cell count in both groups. LPS-treated volunteers had significantly more neutrophils per millilitre of BALF compared with saline-treated subjects (mean $87.9 \pm 89.4 \times 10^{3}$ per $\mathrm{mL}$ versus $0.65 \pm 0.40 \times 10^{3}$ per $\mathrm{mL}$; twosample t-test, $\mathrm{p}=0.039$ ).

Visualisation of the light scatter profiles of cells in BALF by flow cytometry revealed a population of PMLCs, which were distinct from AMs due to their reduced size and granularity (i.e. FSC-A and SSC-A, respectively) (fig. 4). The contribution of PMLCs to total monocyte/macrophage cells in BALF was investigated by selection of $\mathrm{HLA}_{-} \mathrm{DR}^{+} \mathrm{CD} 14^{+}$cells (granulocytes and lymphocytes do not express these markers), which were then defined as AMs or PMLCs based upon their size and granularity. PMLCs were significantly increased in BALF following LPS inhalation, accounting for $46.6 \pm 7.6 \%$ of all monocyte/macrophage cells compared with $5.9 \pm 1.0 \%$ in saline controls $(p=0.0002$, two-sample t-test).

PMLCs were further subdivided into distinct $\mathrm{CD} 14^{++} \mathrm{CD} 16^{-}$and $\mathrm{CD} 14^{++} \mathrm{CD} 16^{+}$subpopulations (reminiscent of classical and intermediate peripheral blood monocytes, respectively) (fig. 4). The most striking observation was of a highly significant increase in the CD $14^{++} \mathrm{CD} 16^{-}$portion of these cells following LPS inhalation, whereas the relative number of $\mathrm{CD} 14^{++} \mathrm{CD} 16^{+}$ PMLCs remained constant in both LPS and saline-treated groups (fig. 5). Therefore, PMLC subpopulations were classed as "inducible" and "resident", respectively. It is noteworthy that very few $\mathrm{CD} 14^{+} \mathrm{CD} 16^{++}$PMLCs (reminiscent of nonclassical peripheral blood monocytes) were observed in BALF from either LPS or saline-treated volunteers.

In contrast to our observation that the proportion of $\mathrm{CD}^{+} \mathrm{CD}^{+} \mathrm{CD}^{2} 5^{+} \mathrm{CD} 127^{-}$Tregs in peripheral blood was unchanged with LPS inhalation, the percentage of Tregs (relative to total $\mathrm{CD}^{+} \mathrm{CD}^{+}$lymphocytes) in BALF at $8 \mathrm{~h}$ post-LPS inhalation was significantly decreased, compared with saline controls ( $3.35 \pm 0.72 \%$ versus $6.8 \pm 0.73 \%$; $p=0.027$ ) (fig. 4 ).

\section{Cytokines in BALF}

Concentrations of IL-6 and MCP-1 were significantly elevated in BALF from LPS-treated volunteers (table 4). Differences in concentrations of IL-8 approached but did not reach significance. Although a precise analysis of TNF- $\alpha$ concentration was not possible, TNF- $\alpha$ was only detectable in BALF from volunteers who had inhaled LPS.

\section{DISCUSSION}

The LPS inhalation model provides controlled conditions in which to study the pulmonary influx of cells in a self-limiting, resolving model of lung inflammation. Our LPS-treated volunteers had demonstrable local and systemic inflammation, as evidenced by a significant neutrophilia in BALF and peripheral blood, and significant rises in the concentration of inflammatory cytokines in BALF. We have developed a reproducible protocol for comprehensive characterisation of surface antigen expression by monocyte subsets, neutrophils and regulatory T-lymphocytes in whole blood and in BALF, by multiparameter flow cytometry.

Flow cytometric analysis of peripheral blood showed that the cell surface phenotype of monocyte subsets and Tregs remained unchanged throughout the time course of LPS inhalation compared to saline controls. Moreover, the ratio of each peripheral blood monocyte subset in whole blood was unchanged with LPS. This study provides an extensive evaluation of monocyte phenotype in healthy human peripheral blood, in line with the recent classification of monocytes into classical, intermediate and nonclassical subpopulations [24]. Our data highlight important differences between the intermediate and nonclassical monocyte subpopulations, which until recently were predominantly analysed as a homogenous $\mathrm{CD}^{+} 6^{+}$population [25]. For example, intermediate monocytes strongly express CD64, CCR2 and CD62L, whereas nonclassical monocytes do not, and, conversely, nonclassical monocytes express the fractalkine receptor, CX3CR1, whereas intermediate monocytes lack this expression.

The surprisingly bland response among the blood leukocyte populations was in striking contrast to the situation observed in the lung where, among the cells retrieved by BAL, we have characterised a pulmonary monocyte-like population and further subcategorised distinct "resident" and "inducible" subpopulations of these cells, for the first time. Monocyte-like cells have been observed in various inflammatory lung conditions [7, 10, 26]. Similarly, a population of monocytes with reduced phagocytic capacity and increased expression of markers of dendritic cell maturation was described in sputum from healthy volunteers following LPS inhalation [27], and a "small sputum macrophage" was reported in patients with COPD [28]. However, 

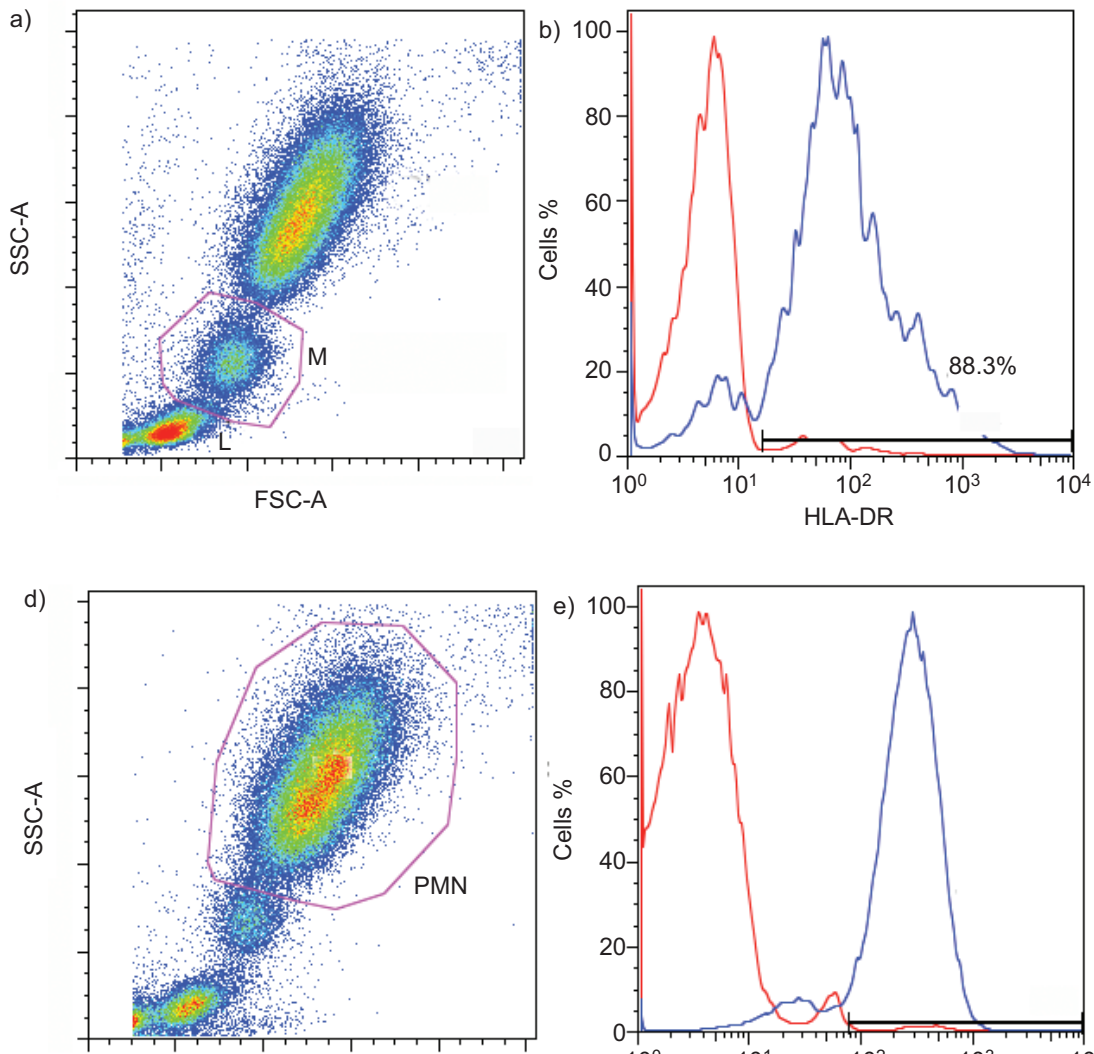

FSC-A

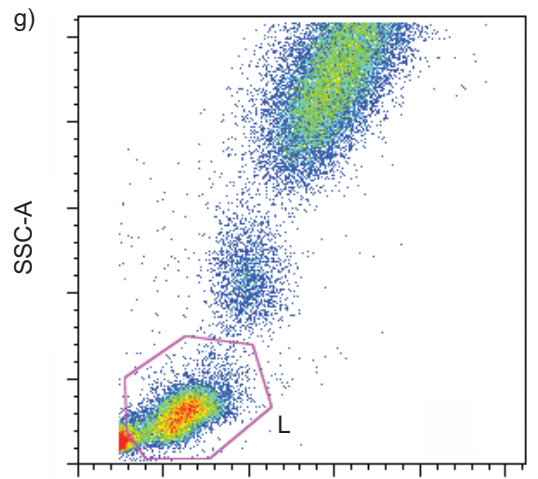

FSC-A
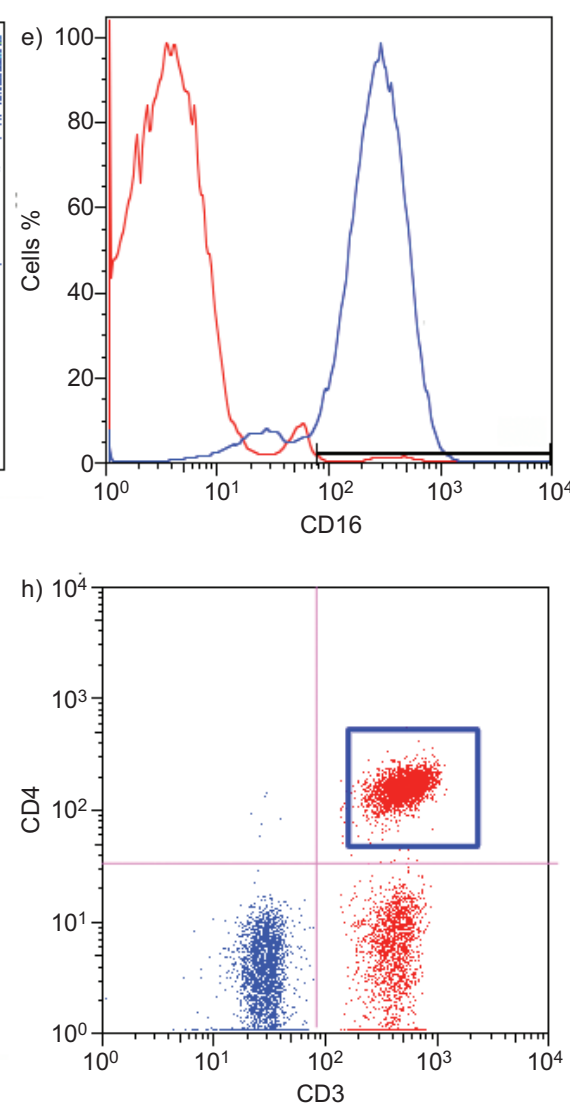
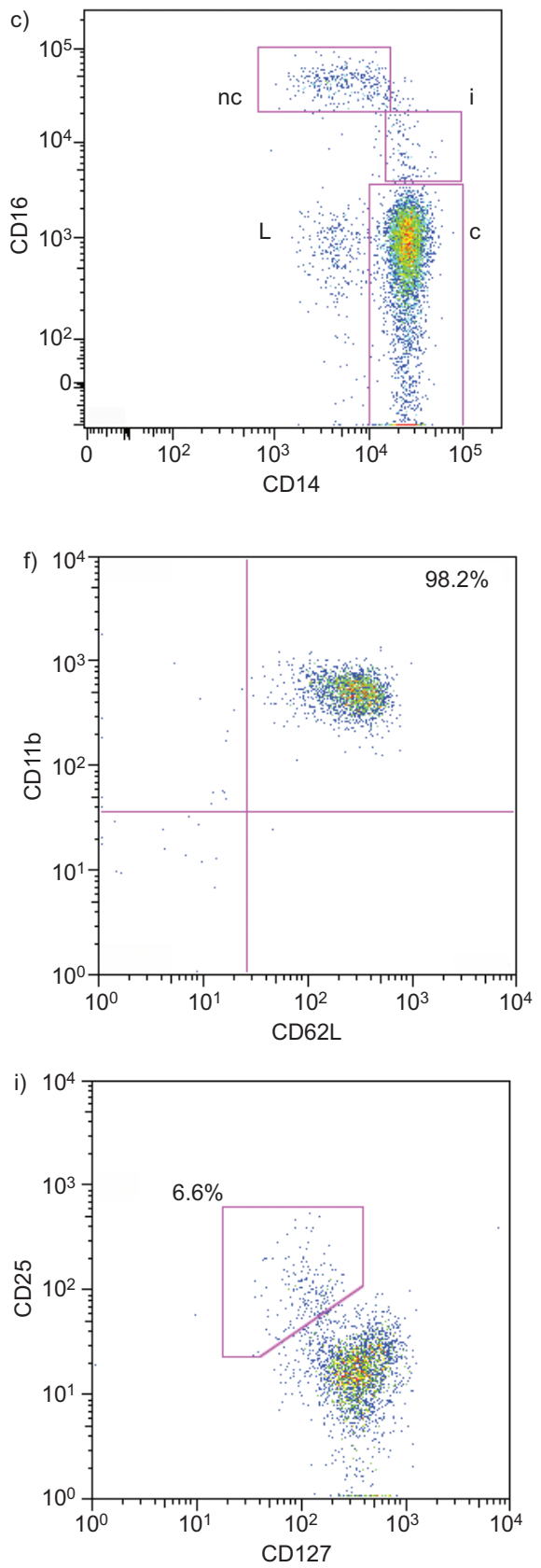

FIGURE 3. Gating strategies for monocyte subpopulations, neutrophils and Tregs in whole blood. a) Monocytes (M) were gated based upon their known light scatter properties on forward scatter-area (FSC-A) versus side scatter-area (SSC-A) dot plots. b) Human leukocyte antigen (HLA)-DR expression was further used to select monocytes and eliminate granulocytes. C) HLA-DR ${ }^{+}$monocytes were then defined as classical (c), intermediate (i) or nonclassical (nc) based upon CD14 and CD16 expression (HLA-DR ${ }^{+}$lymphocytes (L) were not included in the analyses). Polymorphonuclear neutrophils (PMN) were d) gated on FSC-A versus SSC-A dot plots and then e) selected for CD16 expression. f) Changes in expression of CD62L, CD11b (and CD64, not shown) by CD16 ${ }^{+}$PMN were then assessed. Lymphocytes were gated on g) FSC-A versus SSC-A dot plots, and h) $\mathrm{CD}^{+}{ }^{+} \mathrm{CD} 4^{+}$lymphocytes selected. i) The proportion of Tregs $\left(\mathrm{CD}^{+} \mathrm{CD}^{+} \mathrm{CD} 25^{+} \mathrm{CD} 1277^{-}\right)$was calculated as a percentage of $\mathrm{CD} 3^{+} \mathrm{CD} 4^{+}$ lymphocytes using an orthogonal gate.

our data represent the first detailed characterisation of these cells in the context of self-limiting lung inflammation. While we cannot definitively prove that iPMLCs are blood-derived, their rapid appearance in the lung and shared characteristics with "classical" blood monocytes strongly suggest the circulation as the origin. We propose that, during self-limiting lung inflammation, $\mathrm{CD} 14^{++} \mathrm{CD} 16^{-}$ monocytes are rapidly sequestered from the circulation, at a time when blood monocyte populations appear relatively quiescent, and infiltrate the lung in synchrony with neutrophils. Importantly, PMLCs were not believed to be dendritic cells due to their expression of CD11b and high expression of CD14 [20]. We cannot exclude the possibility that $\mathrm{CD} 14^{++} \mathrm{CD} 16^{-}$monocytes may further differentiate in the pulmonary compartment, and this should be the focus of future work. 

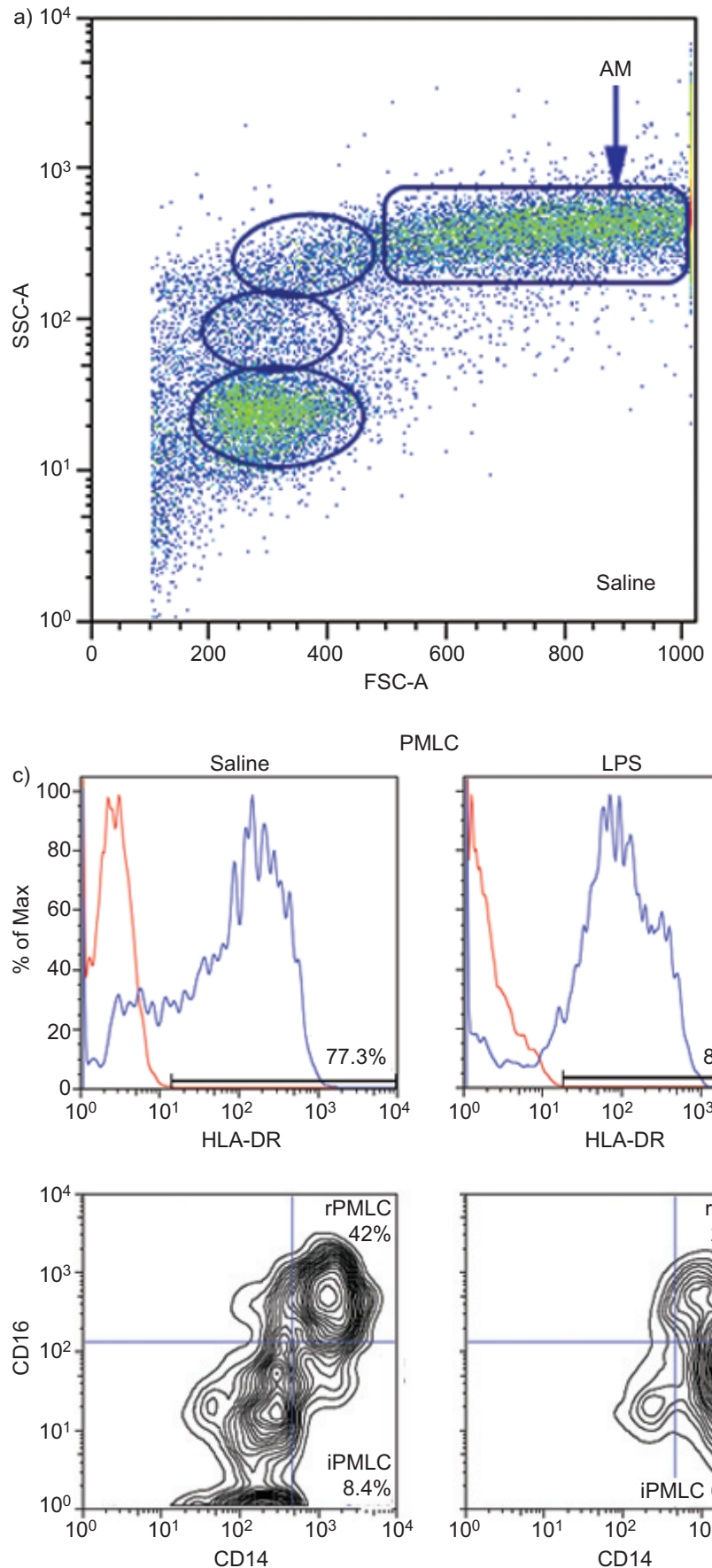

PMLC
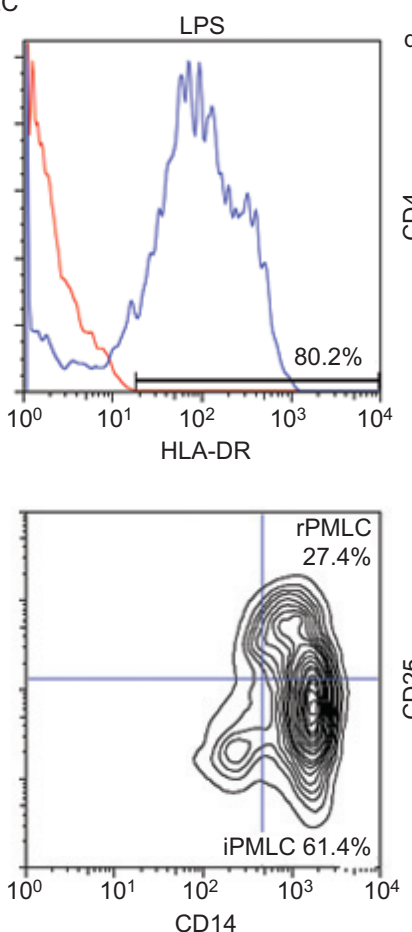
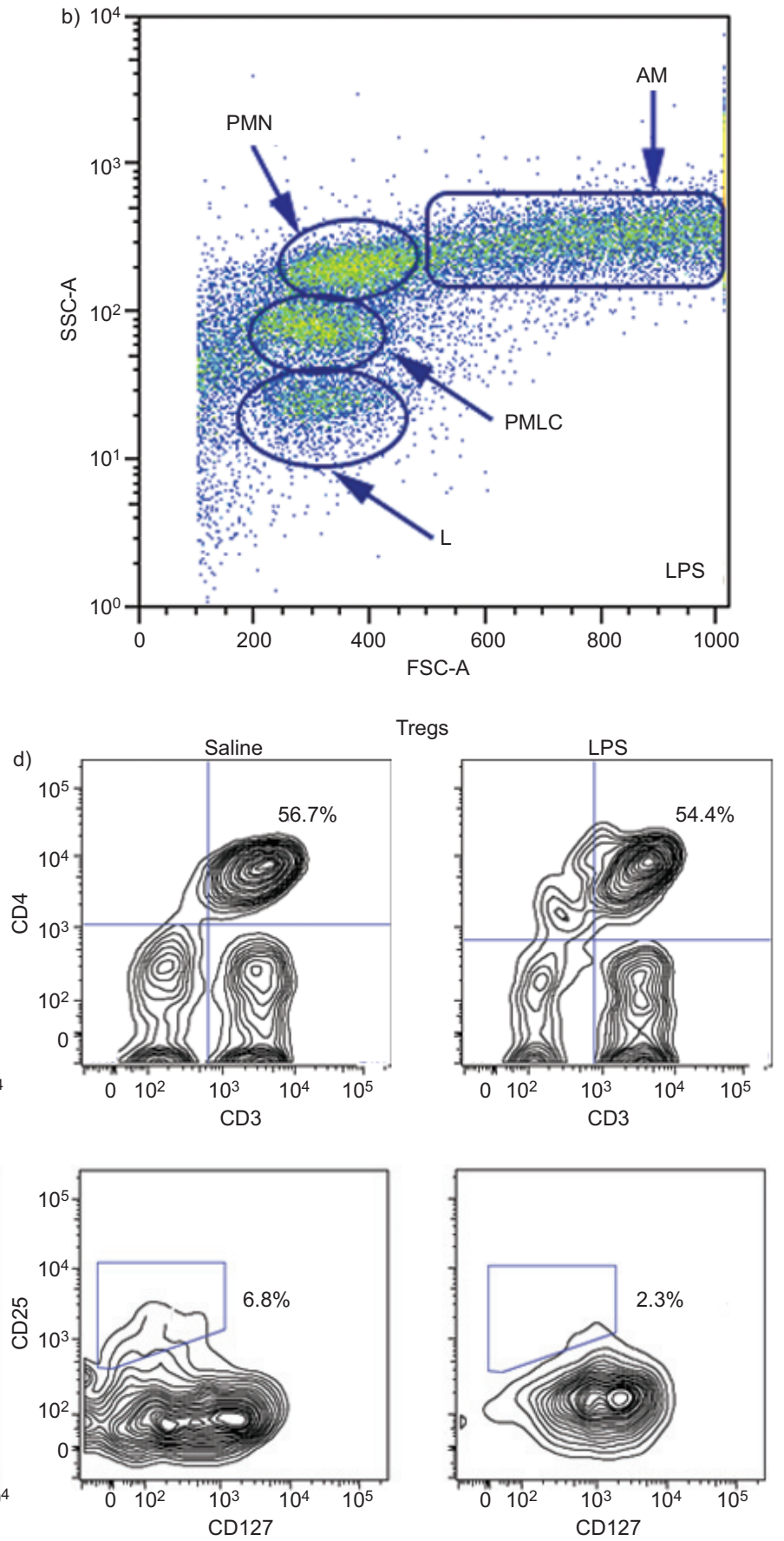

FIGURE 4. Cell populations in bronchoalveolar lavage fluid (BALF) following lipopolysaccharide (LPS) or saline inhalation. Total cellular events in BALF were viewed in forward scatter-area (FSC-A) versus side scatter-area (SSC-A) flow cytometry dot plots. Polymorphonuclear neutrophils (PMN) and pulmonary monocyte-like cells (PMLCs) were significantly increased following b) LPS inhalation compared with a) saline controls, whereas T-cell regulators (Tregs) were significantly decreased following LPS inhalation. c) PMLCs were analysed by selecting human leukocyte antigen (HLA)-DR ${ }^{+}$cells and were then subdivided into inducible (iPMLC; $\mathrm{CD}^{+4^{+}} \mathrm{CD}^{-} 6^{-}$) or resident (rPMLC; $\mathrm{CD} 14^{++} \mathrm{CD} 16^{+}$) subpopulations based upon $\mathrm{CD} 14$ and $\mathrm{CD} 16$ expression. d) $\mathrm{CD}^{+} \mathrm{CD}^{+}$lymphocytes within the lymphocyte gate were further selected and the proportion of $\mathrm{CD}^{+} \mathrm{CD} 4^{+} \mathrm{CD} 25^{+} \mathrm{CD} 127^{-}$Tregs in saline and LPS groups calculated. AM: alveolar macrophages; L: lymphocytes.

In contrast, rPMLCs were constitutively present in the lungs of saline-treated controls, and remained unchanged in number in our model of acute inflammation. We propose that rPMLCs are endogenous to the lung, with a putative role in the maintenance of macrophage homeostasis. This is supported by observations of AMs derived from $\mathrm{CD}_{16}{ }^{+}$, but not CD16 peripheral blood monocytes in the mouse lung [29]. The observed consistency in the number of rPMLCs following LPS inhalation implies that these cells do not undergo a phenotypic switch to become iPMLCs. 


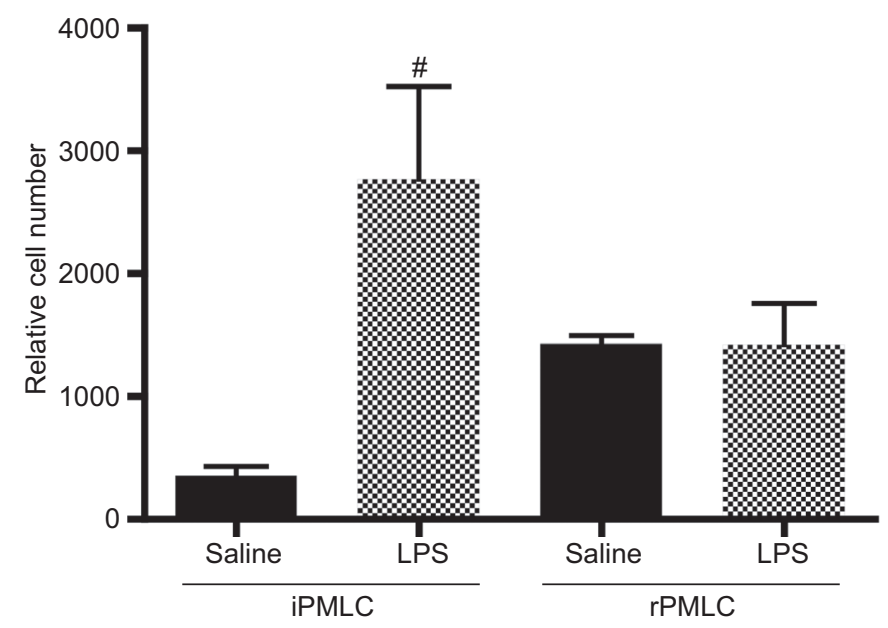

FIGURE 5. Relative numbers of pulmonary monocyte-like cell (PMLC) subpopulations in bronchoalveolar lavage fluid following inhalation of lipopolysaccharide (LPS) or saline. Inducible (i)PMLCs $\left(\mathrm{CD} 14^{++} \mathrm{CD} 16\right)$ were significantly increased following LPS inhalation ( $p=0.0046$; two-sample t-test). No significant change was observed in the numbers of resident (r)PMLCs $\left(\mathrm{CD} 14^{++} \mathrm{CD} 16^{+}\right)$in saline compared with LPS-treated groups. ${ }^{*}: p=0.0046$.

We observed significantly reduced numbers of pulmonary $\mathrm{CD}^{+} \mathrm{CD}^{+} \mathrm{CD} 25^{+} \mathrm{CD} 127^{-}$Tregs at $8 \mathrm{~h}$ post-treatment in our model of LPS-mediated acute lung inflammation, compared with saline controls. Detection of Tregs based upon their positive expression of CD3, CD4 and CD25 and downregulated CD127 expression (rather than expression of the transcription factor, FoxP3), was based upon reports that FoxP3 expression may prove unreliable as a marker of human Tregs as it appears to define a number of functionally heterogeneous populations which differ in their suppressive function and activation status [30,31]. Tregs are proposed to play a role in the resolution of lung inflammation [32], and therefore it seems fitting that these cells are reduced during the acute inflammatory response alongside heightened neutrophilia and increased iPMLCs. It is interesting to speculate that a prolonged and heightened expression of iPMLCs (and reduced

\begin{tabular}{|c|c|c|}
\hline TABLE 4 & $\begin{array}{l}\text { ronchoalveolar lavage fluic } \\
\text { oncentrations }\end{array}$ & cytokine \\
\hline \multirow[t]{2}{*}{ Cytokine } & \multicolumn{2}{|c|}{ Concentration } \\
\hline & Saline & LPS \\
\hline $\mathrm{IL}-6 \mathrm{pg} \cdot \mathrm{mL}^{-1}$ & $64.8(46-144.8)$ & $233.5(153.5-509.8)^{\#}$ \\
\hline $\mathrm{MCP}-1 \mathrm{pg} \cdot \mathrm{mL}^{-1}$ & $\begin{array}{l}\text { All values below lower detection } \\
\text { limit of the assay (15.6) }\end{array}$ & $77.0(42-137)^{\circ}$ \\
\hline $\mathrm{IL}-8 \mathrm{pg} \cdot \mathrm{mL}^{-1}$ & $45(31-53.1)$ & $84.1(52.2-109.4)^{+}$ \\
\hline TNF- $\alpha \mathrm{pg} \cdot \mathrm{mL}^{-1}$ & $\begin{array}{l}\text { All values below lower detection } \\
\text { limit of the assay (15.6) }\end{array}$ & $26.2(15.6-48.7)$ \\
\hline \multicolumn{3}{|c|}{$\begin{array}{l}\text { Date are presented as median (interquartile range). LPS: lipopolysaccharide; IL: } \\
\text { interleukin; MCP: monocyte-chemoattractant protein; TNF: tumour necrosis } \\
\text { factor. }{ }^{*}: \mathrm{p}<0.02 ;{ }^{\circ}: \mathrm{p}<0.005 ;{ }^{+}: \mathrm{p}=0.06 \text {. }\end{array}$} \\
\hline
\end{tabular}

Tregs) may be responsible for disease pathology in conditions of chronic lung inflammation and scarring, such as IPF.

The LPS inhalation model does have some inherent limitations. The LPS is administered over a few minutes only, whereas pathological stimuli for clinical acute inflammation are present for longer. Aetiologies of acute lung injury can be classified as pulmonary or extrapulmonary in origin, and LPS inhalation does not model the latter. In addition, BAL itself may cause a degree of pulmonary inflammation. Our BAL sampled only one segment of the lung and therefore BAL may not be representative of the average inflammation across all lobes and we may not have studied the most informative time-point. However, it is hard to justify repeated bronchoscopies in healthy volunteers. Furthermore, BAL only retrieves cells freely mobilised from the alveolar space and not the interstitium (sampling of which would require at least transbronchial biopsy, which is extremely hard to justify in healthy volunteers). However, despite its limitations, LPS inhalation appears safe and acceptable to healthy volunteers. Furthermore, as we have shown in this study, it provides a consistent method of activating the innate immune response in normal subjects, thus enabling detailed analysis of the cellular fluxes involved in pulmonary inflammation.

In conclusion, our findings add to the limited data relating to pathogenesis in the early stages of acute lung inflammation in humans, and have important and wider implications in defining the relevance of monocyte/macrophage phenotypes in inflammatory lung diseases, and their relationship with neutrophils and Tregs. PMLCs are a major constituent of the cellular repertoire present in the acutely inflamed human lung, and may therefore represent a novel, legitimate target for future therapeutic strategies to treat a range of inflammatory lung diseases.

\section{STATEMENT OF INTEREST}

None declared.

\section{REFERENCES}

1 Rossi AG, Sawatzky DA, Walker A, et al. Cyclin-dependent kinase inhibitors enhance the resolution of inflammation by promoting inflammatory cell apoptosis. Nat Med 2006; 12: 1056-1064.

2 Kreisel D, Nava RG, Li W, et al. In vivo two-photon imaging reveals monocyte-dependent neutrophil extravasation during pulmonary inflammation. Proc Natl Acad Sci USA 2010; 107: 18073-18078.

3 Maus UA, Waelsch K, Kuziel WA, et al. Monocytes are potent facilitators of alveolar neutrophil emigration during lung inflammation: role of the CCL2-CCR2 axis. J Immunol 2003; 170: 3273-3278.

4 Domagala-Kulawik J, Hoser G, Dabrowska M, et al. CD4+/CD25+ cells in systemic inflammation in COPD. Scand J Immunol 2011; 73: 59-65.

5 D'Alessio FR, Tsushima K, Aggarwal NR, et al. CD4+CD25+ Foxp3+ Tregs resolve experimental lung injury in mice and are present in humans with acute lung injury. J Clin Invest 2009; 119: 2898-2913.

6 Hance AJ, Douches S, Winchester RJ, et al. Characterization of mononuclear phagocyte subpopulations in the human lung by using monoclonal antibodies: changes in alveolar macrophage phenotype associated with pulmonary sarcoidosis. J Immunol 1985; 134: 284-292. 
7 Hoogsteden HC, van Dongen JJ, van Hal PT, et al. Phenotype of blood monocytes and alveolar macrophages in interstitial lung disease. Chest 1989; 95: 574-577.

8 Kiemle-Kallee J, Kreipe H, Radzun HJ, et al. Alveolar macrophages in idiopathic pulmonary fibrosis display a more monocyte-like immunophenotype and an increased release of free oxygen radicals. Eur Respir J 1991; 4: 400-406.

9 Wasserman K, Subklewe M, Pothoff G, et al. Expression of surface markers on alveolar macrophages from symptomatic patients with HIV infection as detected by flow cytometry. Chest 1994; 105 : 1324-1334.

10 Rosseau S, Hammerl P, Maus U, et al. Phenotypic characterization of alveolar monocyte recruitment in acute respiratory distress syndrome. Am J Physiol Lung Cell Mol Physiol 2000; 279: L25-L35.

11 Maris NA, de Vos AF, Bresser P, et al. Activation of coagulation and inhibition of fibrinolysis in the lung after inhalation of lipopolysaccharide by healthy volunteers. Thromb Haemost 2005; 93: 1036-1040.

12 Maris NA, de Vos AF, Dessing MC, et al. Antiinflammatory effects of salmeterol after inhalation of lipopolysaccharide by healthy volunteers. Am J Respir Crit Care Med 2005; 172: 878-884.

13 Michel O, Nagy AM, Schroeven M, et al. Dose-response relationship to inhaled endotoxin in normal subjects. Am J Respir Crit Care Med 1997; 156: 1157-1164.

14 Shyamsundar M, McKeown ST, O'Kane CM, et al. Simvastatin decreases lipopolysaccharide-induced pulmonary inflammation in healthy volunteers. Am J Respir Crit Care Med 2009; 179: 1107-1114.

15 Michel O, Duchateau J, Plat G, et al. Blood inflammatory response to inhaled endotoxin in normal subjects. Clin Exp Allergy 1995; 25: 73-79.

16 Sandstrom T, Bjermer L, Rylander R. Lipopolysaccharide (LPS) inhalation in healthy subjects increases neutrophils, lymphocytes and fibronectin levels in bronchoalveolar lavage fluid. Eur Respir J 1992; 5: 992-996.

17 Jagielo PJ, Thorne PS, Watt JL, et al. Grain dust and endotoxin inhalation challenges produce similar inflammatory responses in normal subjects. Chest 1996; 110: 263-270.

18 Conway Morris A, Kefala K, Wilkinson TS, et al. C5a mediates peripheral blood neutrophil dysfunction in critically ill patients. Am J Respir Crit Care Med 2009; 180: 19-28.
19 Shah D, Naciri M, Clee P, et al. NucleoCounter - An efficient technique for the determination of cell number and viability in animal cell culture processes. Cytotechnology 2006; 51: 39-44.

20 Fung E, Esposito L, Todd JA, et al. Multiplexed immunophenotyping of human antigen-presenting cells in whole blood by polychromatic flow cytometry. Nat Protoc 2010; 5: 357-370.

21 Roederer M. Spectral compensation for flow cytometry: visualization artifacts, limitations, and caveats. Cytometry 2001; 45: 194-205.

22 Fazekas de St Groth B, Zhu E, Asad S, et al. Flow cytometric detection of human regulatory T cells. Methods Mol Biol 2011; 707: 263-279.

23 Matthews JN, Altman DG, Campbell MJ, et al. Analysis of serial measurements in medical research. BMJ 1990; 300: 230-235.

24 Ziegler-Heitbrock L, Ancuta P, Crowe S, et al. Nomenclature of monocytes and dendritic cells in blood. Blood 2010; 116: e74-e80.

25 Ancuta P, Liu KY, Misra V, et al. Transcriptional profiling reveals developmental relationship and distinct biological functions of CD16+ and CD16- monocyte subsets. BMC Genomics 2009; 10: 403.

26 Krombach F, Gerlach JT, Padovan C, et al. Characterization and quantification of alveolar monocyte-like cells in human chronic inflammatory lung disease. Eur Respir J 1996; 9: 984-991.

27 Alexis NE, Lay JC, Almond M, et al. Acute LPS inhalation in healthy volunteers induces dendritic cell maturation in vivo. J Allergy Clin Immunol 2005; 115: 345-350.

28 Frankenberger M, Menzel M, Betz R, et al. Characterization of a population of small macrophages in induced sputum of patients with chronic obstructive pulmonary disease and healthy volunteers. Clin Exp Immunol 2004; 138: 507-516.

29 Landsman L, Varol C, Jung S. Distinct differentiation potential of blood monocyte subsets in the lung. J Immunol 2007; 178: 20002007.

30 d'Hennezel E, Yurchenko E, Sgouroudis E, et al. Single-cell analysis of the human $\mathrm{T}$ regulatory population uncovers functional heterogeneity and instability within FOXP3+ cells. J Immunol 2011; 186: 6788-6797.

31 Liu W, Putnam AL, Xu-Yu Z, et al. CD127 expression inversely correlates with FoxP3 and suppressive function of human CD4+ T reg cells. J Exp Med 2006; 203: 1701-1711.

32 Pietropaoli A, Georas SN. Resolving lung injury: a new role for Tregs in controlling the innate immune response. J Clin Invest 2009; 119: 2891-2894. 